\title{
SIGNIFICANCE OF SERUM GAMMA GLUTAMYL TRANSFERASE IN GASTRO- INTESTINAL AND HEPATO BILIARY PANCREATIC MALIGNANCIES WITH SPECIFIC CONSIDERATION TO HEPATIC METASTASIS
}

Naveen Kumar Singh ${ }^{1}$, Sadhna Singh ${ }^{2}$, Rahul Khanna ${ }^{3}$, Tikoo ${ }^{4}$

HOW TO CITE THIS ARTICLE:

Naveen Kumar Singh, Sadhna Singh, Rahul Khanna, Tikoo. "Significance of Serum Gamma Glutamyl Transferasein Gastro-Intestinal and HepatoBiliary Pancreatic Malignancies with Specific Consideration to Hepatic Metastasis". Journal of Evolution of Medical and Dental Sciences 2014; Vol. 3, Issue 05, February 03; Page: 1221-1229, DOI: 10.14260/jemds/2014/1972

ABSTRACT: In this study forty patient's suffering from various gastro intestinal, hepato biliary and pancreatic malignancies specific of serum GGT is studied in patients with hepatic metastasis and also in patients with primary hepatocellular carcinoma along with 20 healthy control. Prospective qualitative study was performed with groups divided based on with or without hepatic metastasis keeping HCC on other end and also into pre and post intervention groups. The result obtained from our study indicates a significant increase of GGT in serum level in patients of HCC. Apart from this, patients suffering from cholangio carcinoma stage IV were also found to be having raised serum GGT level. On the other hand in ca stomach, ca rectum and ca anal canal did not show raised serum Y-GT level but it was raised to some extent in cholangio carcinoma and ca gall bladder cancer particularly in advanced stages like stage III and IV and also even without other evidence, marker's of hepatic metastasis.

These observations showed that raised GGT gives a clear indication for involvement of liver in advanced stage of cancer. Also as per literature and results is in accordance to our study hence serum GGT levels can be well utilized for prognostic purpose before secondary spread into the liver and also very good tool for metastatic detection and recurrence and for follow up in combination with other markers specific to hepatic parenchyma involvement.

INTRODUCTION: The diagnosis of hepatic metastases has therapeutic and prognostic significance in clinical medicine. Various enzyme activity measurements in serum have been used to help detect metastases to the liver. Such determinations have the advantage of being simple ${ }^{[1] S e r u m ~ g a m m a ~}$ glutamyl transferase is found to be specific to liver otherwise also seen to be rasied in pancreatic, renal\& others lesions. This enzyme is well established in hepatic disease both in malignant lesion like primary HCC and secondaries to liver. In the pre cancerous and cancerous phases of hepatic tissues, significant changes occur in GGT specific activities. As a result of the damage of hepatocytes, GGT is released into blood to bring about the rise of their activities in the early phases of cancerization. Moreover, the features of GGT electro chromatography are different from those of normal liver tissues. Hepatic functions in detoxification and biotransformation could be strengthened by enzymatic action which relieves toxic effect of chemical mutagens, carcinogens and other substances and protects normal hepatocytes from the effect of cancer initiating and promoting factors. When the abnormal expression of GGT synthesized genes is controlled, hepato-carcinoma-related GGT is secreted with the characteristics of embryonic liver, which are regarded as the early enzyme marker of hepato-carcinogenesis.[2]. 
GGT exhibits a tissue specific expression that is modified under various physiologic and pathologic conditions, such as development and carcinogenesis. The expression is the highest in embryo livers and decreases rapidly to the lowest after birth. GGT is widely distributed enzyme that has been extensively studied in relation to hepato-carcinogenesis, which is closely related with DNA synthesis and nucleic acid metabolism. Its fundamental causes are the activation of pro oncogenes or the inactivation of tumor suppressor genes initiated by carcinogens. (2)

To evaluate the role of serum gamma glutamyl-transferase we subjected 40patients of gastro intestinal and hepato- biliary malignancies with reference to staging, prognosis and recurrence of malignancies along with 20 control cases (healthy individuals).

AIM: To study the significance of serum gamma glutamyl transferase as marker for staging, prognosis and recurrence of Gastro intestinal, hepato biliary and pancreatic malignancies with specific consideration for hepatic metastsis and primary hepato cellular carcinoma.

MATERIAL AND METHOD: A prospective qualitative study design was applied for observation.

Selection of control: A group of 20 individuals-healthy control who were free from any malignant lesion or any condition which effect serum levels of study enzymes were selected.

Selection of study group: A group of forty patients admitted in surgical division of IMS BHU Varanasi having malignant lesion of hepato-biliary, gastro intestinal and pancreatic area were selected for study purpose for two and half year duration. In all the cases diagnosis was confirmed by histopathology along with imaging. Patient population were divided into three sub groups.

Group A - Local malignant lesion.

Group B - Metastasis to other sites excluding liver.

Group C - Metastasis to other sites including liver.

In these study groups pre-intervention and post intervention levels of study enzyme was measured keeping in mind stages of malignancies in various lesion with special consideration for cases with hepatic metastasis.

Types of malignancies under study were carcinoma stomach, carcinoma gall bladder, peri ampullary carcinoma, cholangio-carcinoma, HCC, colo- rectal carcinoma and ca anal canal. The presence or absence of hepatic metastases was determined by liver scan, liver echogram, biopsy, or some combination of these liver echogram was done in 40 patients, and per cutaneous biopsy or laparotomy in few patients.

Clinical apprisal: Brief notes of case history with examination findings and also operative findings were made as per specification laid down on a proforma. Details of routine and specific investigations, histo- pathology were taken into consideration.

Enzyme study: serum values of the enzymes were determined simultaneously in all forty cases. The values were measured on admission i.e. pre-treatment then after therapeutic interventions. In patients who expired within 24 to 48 hours of surgery or therapeutic intervention, monitoring could not be done. 
Collection of sample: Prior consent was taken from every patients six ml of venous blood from cubital vein by direct vene-puncture, precaution was taken to avoid haemolysis of blood. Sample was allowed to stand at room temperature till serum separates which was centrifuged at $2000 \mathrm{rpm}$ for fifteen min. and stored at 4 degree centigrade in refrigerator.

\section{Pricnciple of estimation of gamma glutamyl transferase and calculation:}

The principle applied is based on reaction

GPNA+Glycylglycin-P-Nitro aniline----------- $\rightarrow+$ - -Gamma-Glutamyl glycyl glycine.

The rate of increase of absorbance due to release of p-nitro aniline is directly proportional to the activity of GGT.

Normal value: $3-20 \mathrm{IU} / \mathrm{ml}$

Serum GGT Conc. In IU/L =OD X 1111 linear upto 180IU/L. There is no interference from other constituents in the serum, method is highly sensitive and specific.

Staging of malignancies were done according to TNM Classification and also treatment was based on the stage of the malignancies with all the three modalities surgery, chemotherapy and radio therapy as applicable to each histological type and tumour sensitivity(both neo adjuvant and adjuvant taken into consideration).

Statistical evaluation: The following procedure was employed to compare the serum levels of enzyme between control group and malignant groups as per stage of the malignancies.

1. For precise data and to establish the variation in groups, parameters like mean and standard deviation calculated.

2. For difference between means, the test of significance was applied. Unpaired ' $t$ ' test for difference between two means i.e., for comparing the activities of serum enzyme of the control group and the different cancer groups.

3. The paired ' $\mathrm{t}$ ' test -null hypothesis was set up.

4. The(M) mean and standard deviation(S.D.) were calculated for different groups separately.

5. The standard error of mean was calculated as per formula SE = S.D.x/ square root of number of observations.

6. The ' $\mathrm{t}$ ' value was calculated $\mathrm{t}=\mathrm{Mx}-\mathrm{My} /$ square root (S.Ex) $\mathrm{x} 2+(\mathrm{S}$.Ey) $\mathrm{x} 2$ along with degrees of freedom, for $t$ was $(n 1+n 2-2)$ where $n 1 n 2$ are the number of observations in control and different cancer groups.

7. After referring to ' $t$ 'distributaion table the probability level $\mathrm{P}$ for null hypothesis was worked out.

8. If $\mathrm{P}$ was more than five percent the mean difference was considerd insignificant. If $\mathrm{P}$ was less than five percent the mean difference was regarded as significant. 


\section{OBSERVATIONS:}

\begin{tabular}{|c|c|c|c|}
\hline S.No. & Age & Sex & GGT (IU/L) \\
\hline 1 & 21 & M & 12 \\
\hline 2 & 33 & M & 15 \\
\hline 3 & 45 & M & 20 \\
\hline 4 & 47 & M & 21 \\
\hline 5 & 32 & M & 16 \\
\hline 6 & 50 & F & 21 \\
\hline 7 & 54 & M & 16 \\
\hline 8 & 62 & M & 13 \\
\hline 9 & 61 & M & 14 \\
\hline 10 & 35 & F & 18 \\
\hline 11 & 51 & F & 16 \\
\hline 12 & 57 & M & 8 \\
\hline 13 & 42 & M & 9 \\
\hline 14 & 43 & M & 12 \\
\hline 15 & 65 & M & 18 \\
\hline 16 & 51 & F & 22 \\
\hline 17 & 39 & M & 17 \\
\hline 18 & 55 & F & 16 \\
\hline 19 & 52 & M & 13 \\
\hline 20 & 65 & M & 12 \\
\hline
\end{tabular}

Table no. 1: Normal value of serum gamma Glutamyl transferase in control group

Mean ofGGT in normal/control group is 1FIVEandS.D.is3.7

\begin{tabular}{|c|c|c|l|c|c|c|}
\hline Caseno & Age & sex & \multicolumn{1}{|c|}{ Disease } & stage & Pre-treatment & Post -treatment \\
\hline 1. & 70 & M & Ca.stomach & III & 22 & 20 \\
\hline 2. & 70 & M & Ca.stomach & II & 65 & 54 \\
\hline 3. & 50 & M & HCC & IV & 202 & 104 \\
\hline 4. & 35 & M & Ca.colon & II & 24 & 22 \\
\hline 5 & 55 & M & Ca.stomach & II & 18 & 16 \\
\hline 6 & 55 & F & Cholangio.ca & IV & 190 & 50 \\
\hline 7 & 58 & M & Ca.stomach & IV & 16 & 16 \\
\hline 8 & 45 & F & Ca.gall bladder & II & 13 & 11.2 \\
\hline 9 & 56 & F & Ca-anal canal & IV & 19 & 17 \\
\hline 10 & 70 & F & Ca.recto-sigmoid & IV & 18 & 17 \\
\hline 11 & 22 & M & Ca.anal canal & IV & 12 & 10.2 \\
\hline 12 & 72 & M & Ca, stomach & II & 14 & 12 \\
\hline 13 & 70 & M & Colangioca & III & 86 & 57 \\
\hline
\end{tabular}


ORIGINAL ARTICLE

\begin{tabular}{|c|c|c|c|c|c|c|}
\hline 14 & 65 & $\mathrm{M}$ & Ca.stomach & III & 12 & 10 \\
\hline 15 & 56 & $M$ & Ca.stomach & III & 17 & 19 \\
\hline 16 & 65 & $\mathrm{~F}$ & Ca.gall bladder & II & 86 & 51 \\
\hline 17 & 75 & $\mathrm{M}$ & Ca.anal canal & III & 17 & 17 \\
\hline 18 & 72 & $\mathrm{M}$ & Ca.stomach & IV & 12 & 10 \\
\hline 19 & 48 & M & $\mathrm{HCC}$ & IV & 220 & 139 \\
\hline 20 & 48 & $\mathrm{M}$ & CholangioCa & II & 94 & 56 \\
\hline 21 & 47 & $\mathrm{M}$ & Ca stomach & II & 19 & 17 \\
\hline 22 & 56 & $\mathrm{M}$ & Ca gall bladder & II & 141 & 97 \\
\hline 23 & 41 & $\mathrm{M}$ & Castomach & III & 16 & 14 \\
\hline 24 & 60 & M & Chalangioca & III & 52 & 35 \\
\hline 25 & 66 & $\mathrm{M}$ & Ca stomach & III & 13 & 30.1 \\
\hline 26 & 58 & $\mathrm{M}$ & Cholangioca & IV & 93 & 49 \\
\hline 27 & 63 & $\mathrm{M}$ & Ca-gall bladder & III & 142 & 91 \\
\hline 28 & 48 & $\mathrm{~F}$ & Ca gall bladder & IV & 187.4 & 155 \\
\hline 29 & 40 & $\mathrm{M}$ & Ca stomach & III & 73.1 & 55 \\
\hline 30 & 68 & M & CholangioCa & II & 146 & 85.5 \\
\hline 31 & 71 & $\mathrm{M}$ & $\mathrm{HCC}$ & III & 230 & 149 \\
\hline 32 & 64 & M & Ca stomach & III & 18 & 14 \\
\hline 33 & 65 & $F$ & Ca gallbladder & III & 94 & 47 \\
\hline 34 & 56 & $\mathrm{M}$ & Ca anal canal & III & 18 & 14 \\
\hline 35 & 67 & $\mathrm{M}$ & $\mathrm{HCC}$ & IV & 211 & 149 \\
\hline $\begin{array}{l}36 \\
37\end{array}$ & 74 & M & Ca stomach & III & 24 & 21 \\
\hline 38 & 64 & $\mathrm{~F}$ & Cagallbladder & IV & 134 & 102 \\
\hline 39 & 59 & $\mathrm{M}$ & Cholangioca & III & 190 & 40 \\
\hline 40 & 72 & $M$ & HCC & IV & 249 & 170 \\
\hline
\end{tabular}




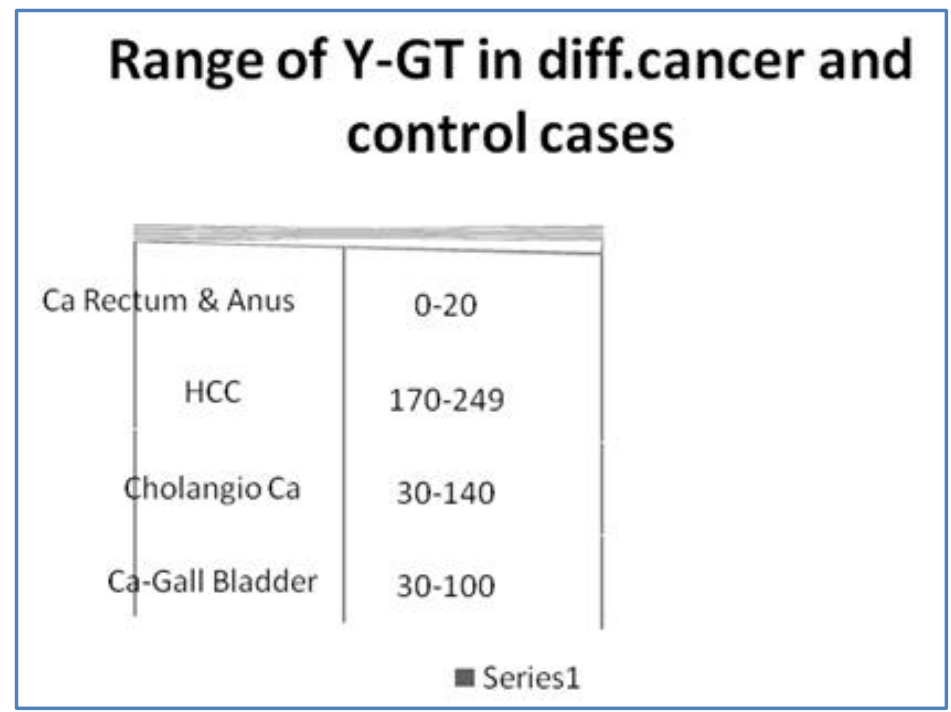

Figure. 1

\begin{tabular}{|l|c|c|c|c|}
\hline \multicolumn{1}{|c|}{ Malignancy } & Mean & SD & T & P \\
\hline Ca stomach & 1.47 & 7.59 & 0.67 & 0.25 \\
\hline Ca gall bladder & 32.98 & 19.01 & 3.87 & $<0.05$ \\
\hline Cholangio ca. & 54.75 & 44.23 & 3.03 & $<0.01$ \\
\hline HCC & 89.50 & 12.00 & 10.52 & $<0.03$ \\
\hline
\end{tabular}

Table 3: Mean and SD of serum Gamma- GT indifferent cancer cases(pre\&post treatment)

\section{RESULTS:}

1. Table no.1- serum Gamma Glutamyltransferase was determined in a group of 20 healthy individuals. The highest and lowest values of enzymes were found to be 22 to 8IU/L respectively the mean value is fifteen and S.D. is 3.7.

2. The level of Gamma -GT has been measured in serum of forty patients. The patients were classified in six groups as already mentioned. The level of serum gamma -GT were found to be as high as 220 to 249IU/L in patients of Hepato cellular carcinoma while in cholangio carcinoma stage III\& IV as well as in few cases of carcinoma gall bladder stage IV is found to be rasied upto $190 \mathrm{IU} / \mathrm{L}$, in one case of carcinoma stomach in stage III it was found raised. Otherwise its level was not riased in ca stomach, ca rectum, ca anal canal but was found to be rasied upto some extent in ca gall bladder \& cholangio carcinoma in advance stages even before establishment of hepatic metastasis. More than fifty percent of the patients showed activity less than twenty fiveIU/L while in few cases of cholangio carcinoma its level is rasied upto 147.IU/L. So in all cases of HCC its value is statistically significant as shown in table no.3. The post therapy value of gamma-GT was assayed after 20 days of treatment and when values compared with pre-treatment values, significant decreases in serum levels was observed in HCC and some cases of stage IV cholangio carcinoma. 
DISCUSSION: Serum enzymes usually reflect the tissue status and differentiation status of the tissue. Most of the enzymes are protein in nature generally in very small concentration in general circulation but increase many fold during malignant transformation of tissue due to destruction and damage of the contiguous normal tissue or systemic effect of neoplasm on normal tissue or by leakage.

The choice of cases in our study was rather random according to availability of the patients in hospital. Based on reports that GGT iso enzymes like alpha feto protein showed increase in case of primary hepato cellular carcinoma before its detection by CT scan even when AFP is negative. Serum GGT ranged between 8to 22 IU/L. Incidence of ca gall bladder is higher in females while ca stomach, cholangio carcinoma and HCC is higher in males.

As described in our study serum GGT level have been found to be highly significant in hepato cellular carcinoma/ primary liver cancer, hepatitis and cirrhosis. GGT found to be increased in patients with HCC compared with benign liver disease. Keeping in view the above studies the present study was undertaken to see the serum Y-GT level in various malignancies.

The result obtained from our study indicates a significant increased in serum level in patients of HCC. Apart from this, patients suffering from cholangio carcinoma stage IV were found to be having raised serum GGT level. On the other hand in ca stomach, ca rectum and ca anal canal did not show raised serum Y-GT level but it was raised to some extent in cholangio carcinoma and ca gall bladder cancer particularly in advanced stages (tableno 3 ).

These observations showed that raised GGT gives a clear indication for involvement of liver in advanced stage of cancer. Also as per litreture and results in accordance in our study serum GGT levels can be well utilized for prognostic purpose before secondary spread into the liver and also very good tool for metastatic detection and recurrence and follow up in combination with other markers specific to hepatic-parenchyma involvement. In a previous study, it was found that blood content of CEA, GPI, GGT, and LD was increased in the serum of patients with cancers of the gastrointestinal tract, breast, and lung $(4,5,6)$; similar increases were also reported in tumor tissue extracts $(7,8)$. Other workers have reported 2.5-fold higher activities of glycolytic enzymes in primary colon carcinomas than in normal colonic mucosae $(8,9)$.

Values for several glycosyl transferases also have been found to be above normal in the sera of patients bearing mammary or colonic carcinomas (10). Moreover, all these enzymes, like CEA, are increased in the serum of patients suffering from various liver diseases; this may be due in part to hepatocellular damage accompanied by compensative hyperplasia. Shonk et al. (8) compared the patterns of glycolytic enzymes in colon carcinomas and hepatomas; they found similar patterns in different colon carcinomas, whereas hepatomas were quite variable. Hilf et al. (3) reported enzyme activities in normal breast tissue, fibrocystic disease, and in infiltrating ductal carcinoma. GPI activity in fibrocystic disease was threefold that in normal breast tissue, and eight- to10-fold higher than normal in breast-cancer tissue.

Because combined data on CEA, GPI, GGT, and LD are becoming of increased clinical interest in monitoring cancer patients, I undertook the present study. The observation that values for these substances are highest in colon cancer metastatic to the liver supports clinical evidence that these markers are of greatest use in reflecting disease activity in patients with liver metestases $(5,6)$. These markers have also been found to be above-normal in the sera of jaundiced patients, alcoholics, and patients with other benign liver diseases $(6,11)$. However, aqueous tissue extracts of primary colon cancers have increased values for these markers, and so such increases in cancer patients may better 
reflect activity in malignant tissues than liver malfunction. Nevertheless, in some patients with the post treatment serum Y-GT level assayed on day 2 indicate an abrupt decrease in its level in HCC and few cases of cholangio carcinomastage IV but in ca gall bladder no significant decrease level was found which can be attributed to non responsiveness to the treatment modalities applied.

Although further studies in this regard are necessary to warrant any definite conclusion and also if other enzymes like LDH, AFP, SGPT and SGOT if combinedly analysed can be considerd as better prognostic marker and help in pro per-interventions for different stages of malignancies of heptobiliary and GI malignancies.

\section{REFERENCES:}

1. Kim K. Noe, Yasmineh G. Walid, Freier F. Esther, Goldman I Anne et al. Clinical Chem. 1977;(23/11): 2034-2038 .

2. Yao DF, Dong ZZ, Yao DB, et al. Abnormal expression of hepatoma-derived gamma glutamyl transferase subtyping and its early alteration for carcinogenesis of hepatocytes. Hepatobiliary Pancreat DisInt 2004;3:564-570.

3. Chao-T.C., Wang-C.S., Jeng-L.B., Jan-YY. Journal of Surg-Oncology. Jan1996;1(1):45-49.

4. Cooper E. H., Turner R., Steele L., et al. The contribution of serum enzymes and carcino embryonic antigen to the early diagnosis of metastatic cob-rectal cancer.Br. J. Cancer 1975; 31: 111-117.

5. Munjal D., Chawla P., Lokich J.J., and Zamcheck N. Carcino embryonic antigen and phosphor hexose isomerase, gamma-glutamyl tranpeptidase and lactate dehydrogenase levels in patients with and without liver metastases. Cancer1976; 37: 1800-1807 .

6. Schwartz M. K. Enzymes in cancer. Clin. Chem. 1973;19: 10-22.

7. Munjal D., and Goldenberg D. M. Quantitation of carcino embryonic antigen (CEA), glucose phosphate isomerase .GPI, 1812.

8. Shonk C. E., Arison R. N., Koven B. J., et al. Enzyme patterns in human tissues. III. Glycolytic enzymes in normal and malignant tissues of the colon and rectum. Cancer Res. 1965;25: 206213.

9. Schwartz M. A, West M., Walsh W. S., and Zimmerman H. J. Serum enzymes in disease. VIII. Glycolytic and oxidative enzymes and transaminases in patients with gastrointestinal carcinoma. Cancer1962;15: 346-353 .

10. Weiser M. W., Podolsky D. K. and Isselbacher K. J. Cancer associated isoenzyme of serum galactosyltransferase (polyacrylamidegel electrophoresis). Proc. Natl. Acad. Sci. USA 1976;73: 1319-1322. 


\section{ORIGINAL ARTICLE}

\section{AUTHORS:}

1. Naveen Kumar Singh

2. Sadhna Singh

3. Rahul Khanna

4. Tikoo

\section{PARTICULARS OF CONTRIBUTORS:}

1. Associate Professor, Department of Surgery, SGRRIM \& HS, SMI Hospital, Dehradun.

2. Assistant Professor, Department of Community Medicine, SGRRIM \& HS, SMI Hospital, Dehradun.

3. Professor \& HOD, Department of Surgery, IMS, BHU, Varanasi.

4. Senior Resident, Department of Biochemistry, IMS, BHU, Varanasi.

\section{NAME ADDRESS EMAIL ID OF THE} CORRESPONDING AUTHOR:

Dr. Naveen Kumar Singh, Associate Professor, Department of Surgery, SGRRIM \& HS, SMI Hospital, Dehradun.

House No. G-15, SGRRIMHS,

E-mail: dr.naveen.s@gmail.com

Date of Submission: 23/01/2014.

Date of Peer Review: 24/01/2014.

Date of Acceptance: 27/01/2014.

Date of Publishing: 29/01/2014. 